\title{
ANÁLISIS DEL RÉGIMEN DE CORRELATIVIDADES DE INGENIERÍA EN AGRIMENSURA MEDIANTE CPM
}

Caputo, L.N.(*)1 Bordón, Paula D.(*)2, Ferrari, Elvira E. ${ }^{(*) 3}$

Resumen: Este trabajo tiene como objetivo analizar el régimen de correlatividades del plan de estudios de Ingeniería en Agrimensura mediante programación por camino crítico, a fin de determinar si su rigidez podría ser un factor que favorece el retraso de los estudiantes en egresar. De los resultados obtenidos, se concluye que, si bien el régimen es lo suficientemente flexible en cuanto a la aprobación de las asignaturas, no lo es en igual medida en lo que respecta al cursado y regularización de las mismas.

Introducción: Tomando como antecedentes trabajos similares realizados por el Grupo de Matemática Aplicada a la Investigación Educativa [1], [2], [3], [4], el presente trabajo consiste en la determinación de la duración mínima de la carrera Ingeniería en Agrimensura de la FACENA, por el método de programación por camino crítico (CPM), así como también de aquellas asignaturas que — de retrasarse su aprobación — implicarían una mayor duración de la carrera.

Es evidente que, en la práctica, en todas las carreras la duración prescripta por el plan de estudios (a la que a partir de ahora denominaremos "duración teórica”), no coincide con el tiempo requerido por los estudiantes para graduarse ("duración real”), afirmación que ha sido validada para las carreras de Profesorado en Matemática [5], de Programador Universitario de Aplicaciones (título intermedio de la $\mathrm{Li}^{-}$ cenciatura en Sistemas) [6], Agrimensura [7] y los Ciclos Básicos de las carreras Ingeniería en Electrónica e Ingeniería Eléctrica [8] y de la Licenciatura en Ciencias Biológicas [9] de la FACENA.

Este problema de prolongados tiempos de egresos, se ve favorecido, por un alto nivel de desgranamiento y de la dificultad de regularizar [10], [11], [12] y aprobar [13] las distintas asignaturas, principalmente, las de los dos primeros años.

Al analizar el desempeño de los estudiantes de Ingeniería en Electrónica por el método de componentes principales, Avalos et al [14] comprobaron que el primer factor que determina la duración del Ciclo Básico de dicha carrera es el régimen de correlatividades de su plan de estudios.

(*) Facultad de Ciencias Exactas y Naturales y Agrimensura. Universidad Nacional del Nordeste. Avda. Libertad 5450. 3400. Corrientes.

1- Especialista en Docencia Universitaria; proflcaputo@gmail.com

2- Profesora de Matemática; paulabordon85@gmail.com

3- Especialista en Docencia Universitaria; elviraeferrari@yahoo.com.ar 
Por ello, en este trabajo, hemos realizado un análisis del régimen de correlatividades del plan de estudios, a fin de determinar si su rigidez podría ser uno de los factores que produce el atraso de los estudiantes. A tal fin, hemos aislado el problema de otros factores (tales como situación socio-económica de los estudiantes, dificultades de aprendizaje, normativa institucional, obstáculos epistemológicos, etc.), considerando que la duración de la carrera es sólo consecuencia de la secuenciación de los contenidos, es decir, del régimen de correlatividades.

Nuestro objetivo es, mediante los resultados y conclusiones a que arribemos en este trabajo, contribuir a la evaluación del plan de estudios de la carrera y, en consecuencia, al proceso de acreditación de la misma, que se pondrá en marcha en corto plazo.

Contextualización del problema: Breve descripción de la carrera:

La carrera en estudio cuenta con una duración teórica de 5 años.

Su plan de estudios está organizado por asignaturas: 33 cuatrimestrales, 1 anual, un examen de idioma (Inglés Técnico) y dos espacios curriculares de formación profesional — anuales - denominados Práctica Profesional Supervisada y Trabajo Final. De las asignaturas cuatrimestrales 2 tienen carácter optativo, siendo todas las demás (incluidos la anual, el examen y los espacios de formación profesional) de carácter obligatorio para la obtención del título de Ingeniero.

En el marco de la Optativa I, los estudiantes pueden elegir entre:

a) Elementos de Edificios. b) Información Rural y Agrología

En cambio, la Optativa II contempla 3 posibilidades:

a) Levantamientos Hidrográficos

b) Epistemología y Metodología de la Investigación

c) Microgeodesia

A su vez, las 34 asignaturas de la carrera se agrupan en bloques temáticos: Ciencias Básicas, Tecnologías Básicas, Tecnologías Aplicadas y Complementarias, de lo que se da cuentas en la Tabla 1.

\section{Metodología:}

El método de programación por camino crítico (CPM) se utiliza para la programación y control de proyectos. Los proyectos que pueden analizarse mediante este método están constituidos por una serie de tareas o actividades que tienen asociada una duración (o tiempo de ejecución) y entre las cuales existe una relación de orden parcial (llamada relación de precedencia) tal que, si dos tareas no están relacionadas directa o indirectamente entre sí es porque pueden realizarse simultáneamente.

Es importante señalar que, en este tipo de proyectos, sólo es posible iniciar la ejecución de una cierta tarea si se ha finalizado la ejecución de todas sus predecesoras.

Asimismo, estos proyectos contienen actividades tales que, de retrasarse su ejecución, se incrementa el tiempo de ejecución del proyecto. Dichas tareas son las denominadas tareas críticas y tienen holguras o márgenes totales nulos (la holgura total de cada tarea es el tiempo que ésta puede retrasarse sin que ello afecte la duración total del proyecto). 


\begin{tabular}{|c|c|c|c|}
\hline Bloque Temático & Asignatura & Año & Cuatrimestre \\
\hline \multirow{11}{*}{$\begin{array}{l}\text { CIENCIAS } \\
\text { BASICAS }\end{array}$} & Algebra y Geometría Analítica & \multirow{7}{*}{1} & \multirow{3}{*}{1} \\
\hline & Cálculo Diferencial e Integral I & & \\
\hline & Química General & & \\
\hline & Cálculo Diferencial e Integral II & & \multirow{4}{*}{2} \\
\hline & Mecánica, Óptica y Sonido & & \\
\hline & Informática & & \\
\hline & Sistemas de Representación & & \\
\hline & Estadística y Análisis de Datos & \multirow{3}{*}{2} & \multirow{3}{*}{1} \\
\hline & Trigonometría & & \\
\hline & Electricidad, Magnetismo y Calor & & \\
\hline & Análisis Matemático & 3 & 1 \\
\hline \multirow{6}{*}{$\begin{array}{l}\text { TECNOLOGIAS } \\
\text { BASICAS }\end{array}$} & Introducción a la Ing. en Agrim. & \multirow{5}{*}{2} & $\mathbf{l}$ \\
\hline & Agrimensura Legal I & & \multirow{4}{*}{2} \\
\hline & Geografia Física y Geomorfología & & \\
\hline & Teoría de Errores y Comp. & & \\
\hline & Topografia I & & \\
\hline & Dibujo Topográfico Cartográfico & 3 & 1 \\
\hline \multirow{13}{*}{$\begin{array}{l}\text { TECNOLOGIAS } \\
\text { APLICADAS }\end{array}$} & Fotointerpretación y Teledirección & \multirow{4}{*}{3} & 1 y 2 \\
\hline & Topografia II & & 1 \\
\hline & Topografia III & & \multirow{2}{*}{2} \\
\hline & Agrimensura Legal II & & \\
\hline & Catastro & \multirow{8}{*}{4} & \multirow{4}{*}{1} \\
\hline & Fotogrametría & & \\
\hline & Ordenamiento Territorial & & \\
\hline & $\begin{array}{lll}\begin{array}{l}\text { Geodesia } \\
\text { Matemática }\end{array} & \text { Astronómica } & \text { y } \\
\end{array}$ & & \\
\hline & Valuaciones Inmobiliarias & & \multirow{4}{*}{2} \\
\hline & Geodesia Física y Global & & \\
\hline & Cartografia & & \\
\hline & Mensura & & \\
\hline & Sistemas de Información Territorial & 5 & 1 \\
\hline \multirow{4}{*}{ COMPLEMENTARIAS } & Optativa I & \multirow{2}{*}{3} & 1 \\
\hline & Economía y Gestión Empresarial & & 2 \\
\hline & Mediciones Especiales & \multirow{2}{*}{5} & 1 \\
\hline & Optativa II & & 2 \\
\hline
\end{tabular}

Tabla 1: Bloques temáticos, asignaturas y su ubicación en el plan de estudios

Es usual representar un proyecto me- plano $\mathrm{V}$ (cada elemento de $\mathrm{V}$ es un vértice diante un grafo dirigido (o dígrafo) conexo del grafo) y un conjunto de pares ordenados y simple es decir, un conjunto de puntos del de elementos de $V$, cada uno de los cuales se 
denomina arco del dígrafo, en el cual no existen vértices aislados, bucles ni lados paralelos.

Un camino del grafo constituido en su totalidad por actividades críticas se denomina ruta crítica, camino crítico o camino de valuación máxima. Un mismo proyecto puede contener más de una ruta crítica. En consecuencia, la duración mínima del proyecto está dada por la suma de las duraciones de las actividades de un camino crítico del proyecto. [15]

Al representar la ejecución de un proyecto mediante el dígrafo mencionado, a fin de respetar el orden de ejecución de las tareas, suele ser necesario introducir actividades ficticias o dummys, de duraciones nulas y que se representan con líneas de puntos. A fin de minimizar el número de estas tareas y facilitar la visualización de los resultados obtenidos sobre el grafo, es conveniente construirlo, utilizando sus vértices para representar las actividades y los arcos para señalar la relación de orden existente entre ellas (Método de Roy o de los potenciales).

En estos casos, dado un proyecto de $\mathbf{n} \in \mathbb{N}$ actividades, es usual introducir dos dummys - que usualmente se denotan con 0 y $n+1$ - que representan, respectivamente, las actividades de iniciar y de finalizar la ejecución del proyecto.

Una vez estimada la duración de cada actividad $\left(\mathrm{d}_{0}, \mathrm{~d}_{1}, \ldots, \mathrm{d}_{\mathrm{n}+1}\right)$ y establecidas con precisión todas las tareas que preceden a i (conjunto $\mathrm{P}_{\mathrm{i}}$ ) y todas las que la suceden (conjunto $\mathrm{S}_{\mathrm{i}}$ ) es factible construir el grafo mencionado en el párrafo anterior.

Así, de acuerdo a lo propuesto por Pérez Mackeprang et al [15], para determinar los tareas críticas del proyecto, es necesario establecer - para cada actividad i del proyecto, con $0 \leq \mathrm{i} \leq \mathrm{n}+1-$ los momentos más próximos de inicio y de finalización de ejecución de la tarea i, a los que denotaremos con MPI y MPF ${ }_{i}$, respectivamente. De la misma manera, pueden calcularse los momentos más tardíos de inicio y finalización de la actividad i, denotados, respectivamente, con $\mathrm{MTI}_{\mathrm{i}}$ y MTF $\mathrm{M}_{\mathrm{i}}$. Para hallar los momentos más próximos se recorre el grafo de izquierda a derecha (desde el vértice 0 al $n+1)$ y para calcular los más tardíos se lo recorre en sentido contrario.

Es evidente que $\mathrm{MPI}_{0}=0$. En cambio, para la actividad $\mathbf{i}$, con $1 \leq \mathbf{i} \leq \mathrm{n}+1$, se tiene que $\mathrm{MPI}_{\mathrm{i}}=\operatorname{máx}\left\{\mathrm{MPFj} / \mathrm{j} \in \mathrm{P}_{\mathrm{i}}\right\}$. En efecto, el momento más próximo en que puede empezar a ejecutarse la actividad i es el momento más cercano en el tiempo en que todas sus predecesoras han sido finalizadas. Asimismo, es sencillo comprender que $M P F_{i}=M P I_{i}+d_{i}$.

Como el objetivo del método es hallar la duración mínima de realización del proyecto, es obvio que el $\mathrm{MTF}_{\mathrm{n}}+1=\mathrm{MPF}_{\mathrm{n}+1}$ $={ }^{1} \mathrm{MPI}_{\mathrm{n}+1}=\mathrm{MTI}_{\mathrm{n}+1}$. Así pues, $\mathrm{MTF}_{\mathrm{n}+1}=$ $\mathrm{MPF}_{n+1}=\mathrm{MPI}_{\mathrm{n}+1}=\mathrm{MTI}_{\mathrm{n}+1}$ es la duración mínima del proyecto.

Para calcular los momentos más tardíos de inicio y de finalización de una actividad i, con $0 \leq \mathbf{i} \leq \mathrm{n}$, se utilizan las ecuaciones:

1- Por ser $\mathrm{dn}+1=0$ 
$\mathrm{MTF}_{\mathrm{i}}=\operatorname{mín}\left\{\mathrm{MTI}_{\mathrm{j}} / \mathrm{j} \in \mathrm{S}_{\mathrm{i}}\right\}$ y MTI $=$ $\mathrm{MTF}_{\mathrm{i}}-\mathrm{d}_{\mathrm{i}}$.

Ahora bien, hemos dicho que una actividad i es crítica si su margen total, holgura o slacks es nulo, de donde sus momentos más tardío y más próximo de finalización coinciden. De ahí que para calcular la holgura de una tarea i, utilizaremos la ecuación $\mathrm{MT}_{\mathrm{i}}=\mathrm{MTF}_{\mathrm{i}}-\mathrm{MPF}_{\mathrm{i}}=0$.

En este trabajo, se considera que el plan de estudios de la carrera es el proyecto a analizar. Las actividades del proyecto están dadas por las tareas de aprobar cada una de las asignaturas del plan, el examen de traducción y los dos espacios curriculares de formación profesional; la relación de precedencia entre las tareas está determinada por el régimen de correlatividades.

Para determinar la duración de cada actividad, se establecen los siguientes supuestos, adecuándolos a los expuestos por Caputo en 2007 [16]:

- La unidad de tiempo elegida para determinar la duración de cada tarea es el día.

- La fecha de ingreso de cada estudiante es el 1 de marzo del año en que se inicia en los estudios.

- Cada año lectivo finaliza el 31 de diciembre. En consecuencia, el primer año de estudios tiene una duración de 10 meses calendario (306 días), y los posteriores de 12 meses (365 días).

-Asimismo, se establece que el primer cuatrimestre de cada año lectivo se inicia el 1 de marzo y finaliza el 30 de junio, mientras que el segundo comienza el 1 de agosto y termina el 30 de noviembre.

- Se considera que los estudiantes no rinden examen de una asignatura en condición de libres sin haberla cursado, por lo cual al tiempo necesario para haberla cursado se le suman 31 días para aprobarla, ya que se supone que la actividad de aprobación no consiste sólo en presentarse ante la mesa examinadora, sino que también contempla el tiempo necesario para la preparación del alumno a tal fin.

- El tiempo requerido para aprobar una asignatura sin requisito de correlatividades previas, está dada por la diferencia entre la fecha de aprobación de dicha asignatura y la fecha de ingreso. Este supuesto se mantiene, aunque la asignatura en cuestión pertenezca a un año posterior al primer año de estudios. Luego, si se trata de una asignatura correspondiente al primer cuatrimestre, sin requisitos previos de regularidad, su duración está dada por: 31jul -1 -marzo $+1=153$. Si, en las mismas condiciones corresponde al segundo cuatrimestre, su duración es 31- jul - 1-marzo $+1=306$. Introducción a la Ingeniería en Agrimensura (6) es una asignatura en las condiciones antes mencionadas, pero como su régimen de acreditación es por promoción, sin examen final, se estima su duración como sigue: $\mathrm{d}_{6}=30$-jun -1 -mar$z o+1=122$.

- Si una asignatura i corresponde al segundo cuatrimestre de cualquiera de los años de estudios, y su correlativa más próxima es una asignatura correspondiente al mismo año, su duración es: $\mathrm{d}_{\mathrm{i}}=31$-dic 1 -ag $+1=153$. Las asignaturas Agrimensura Legal I (13) y Cartografía (32), cumplen la condición antes enunciada, sin embargo, 
como ambas son promocionales, sin examen final, se tiene que:

$\mathrm{d} 13=\mathrm{d} 32=30-$ nov $-1-\mathrm{ag}+1=122$

- Si bien Caputo (2007) aconseja introducir relajaciones para modelar los recesos académicos de verano, en este trabajo - para simplificar el modelo- dichos intervalos de tiempo (59 días) se suman a la duración de las tareas de aprobación cuyas ejecuciones se inician al término de dicho receso.

- A partir del segundo año de estudios, si una asignatura i pertenece al primer cuatrimestre y su correlativa más próxima es una asignatura del segundo cuatrimestre del año anterior, resulta: $d_{i}=31$-jul -1 -enero + $1=212$. En el caso de Dibujo Topográfico y Cartográfico (17) se tiene que $\mathrm{d}_{17}=30$-jun - 1-enero $+1=181$, por ser promocional.

- A partir del segundo año de estudios, si una asignatura pertenece al primer (segundo) cuatrimestre y su correlativa más próxima es una asignatura del primer (segundo) cuatrimestre del año anterior, su duración es de 365 días. Algo similar sucede con Fotointerpretación y Teledetección (19) y los dos espacios curriculares (Práctica Profesional Supervisada y Elaboración del Trabajo Final), por ser anuales.

- Como para aprobar Prueba de Traducción Técnica (Inglés) no se exigen correlatividades previas se considera que la correspondiente tarea de aprobación comienza en el momento de inicio de la carrera, pero - como se estima que se requieren conocimientos específicos para aprobarla y dicha aprobación se exige para cursar (y, en consecuencia aprobar) las asignaturas del tercer año de la carrera- se considera que dicho examen se da el 31 de julio del segundo año de estudios, por lo cual la duración de la actividad correspondiente es de 518 días.

- Para la defensa del Trabajo Final, se exige haber aprobado todas las asignaturas. Entonces, para que dicha defensa se realice en época de exámenes (Febrero), se le asigna a esta tarea una duración de 45 días en lugar de los 31 días asignados a las asignaturas. Por tal motivo, la tarea de aprobar el Trabajo Final se la ha desdoblado en 2: la actividad 37 consiste en elaborarlo y la 38 en defenderlo y aprobarlo. Sus duraciones son de 365 y 45 días, respectivamente.

Hasta el momento, se ha hablado de un proyecto tal que, para que pueda considerarse que su ejecución ha finalizado, debe haberse ejecutado todas y cada una de las actividades que lo forman. En dicho caso, el proyecto se modela mediante un grafo denominado red de actividades ciertas (RAC). Sin embargo, la inclusión de dos asignaturas optativas tales que una cuenta con dos opciones y la otra con 3, hace apropiado el uso de una "red de actividades estocásticas” (RAE) para modelar el plan de estudios de la carrera, las cuales se utilizan en proyectos en los cuales existen elementos aleatorios que deben ser incluidos en la definición de la red o grafo [17].

Sin embargo, en este trabajo las asignaturas optativas se incluyeron sin hacer referencia a las opciones de cada una de ellas, pues el escaso número de alumnos 
que hasta el momento han cursado y/o los mismos requisitos de correlatividades, aprobado dichas asignaturas no permi- por lo cual el denotarlas como Optativa I te el cálculo probabilístico que el uso de y Optativa II no producirá ningún tipo de una RAE supone. Cabe señalar que todas ambigüedades respecto a su duración, prelas opciones de cada optativa se rigen por decesoras y momentos de ejecución.

\begin{tabular}{|c|c|c|c|}
\hline Tarea & Aprobar ... & Precedencias & $\begin{array}{c}\text { Duración } \\
\text { (Días) }\end{array}$ \\
\hline 1 & Algebra y Geometría Analítica & ------ & 153 \\
\hline 2 & Cálculo Diferencial e Integral I (CDI1) & ------ & 153 \\
\hline 3 & Química General & 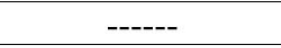 & 153 \\
\hline 4 & Informática & ------ & 306 \\
\hline 5 & Sistema de Representación (S. de R.) & ------ & 306 \\
\hline 6 & Introducción a la Ingeniería en Agrimensura & ------ & 122 \\
\hline 7 & Trigonometría & ------ & 153 \\
\hline 8 & Prueba de Traducción Técnica & ------ & 518 \\
\hline 9 & Cálculo Diferencial e Integral II & 2 & 153 \\
\hline 10 & Mecánica, Óptica y Sonido & 1,2 & 153 \\
\hline 11 & Electricidad, Magnetismo y Calor & 10 & 212 \\
\hline 12 & Estadística y Análisis de Datos & 1,9 & 212 \\
\hline 13 & Agrimensura Legal I & 6 & 122 \\
\hline 14 & Topografía I & $5,7,10$ & 365 \\
\hline 15 & Geografía Física y Geomorfología & 3 & 153 \\
\hline 16 & Teoría de Errores y Compensación & 4,12 & 153 \\
\hline 17 & Dibujo Topográfico y Cartográfico & 8,14 & 181 \\
\hline 18 & Topografía II & 8,14 & 212 \\
\hline 19 & Fotointerpretación y Teledetección & $8,11,15$ & 365 \\
\hline 20 & Análisis Matemático & 8,16 & 212 \\
\hline 21 & Economía y Gestión Empresarial & $4,8,12$ & 153 \\
\hline 22 & Topografía III & 17,18 & 153 \\
\hline 23 & Agrimensura Legal II & $8,13,14$ & 212 \\
\hline 24 & Optativa I: & $5,8,15$ & 365 \\
\hline 25 & Fotogrametría & 19,22, & 212 \\
\hline 26 & Geodesia Astronómica y Matemática (GAM) & 20,22 & 212 \\
\hline 27 & Catastro & 21,23 & 212 \\
\hline 28 & Ordenamiento Territorial & $14,19,21$ & 212 \\
\hline 29 & Geodesia Física y Global & 25,26 & 153 \\
\hline 30 & Mensura & 27,28 & 153 \\
\hline 31 & Valuaciones Inmobiliarias & 27 & 153 \\
\hline 32 & Cartografía & $17,25,26$ & 122 \\
\hline 33 & Mediciones Especiales & 29 & 212 \\
\hline 34 & Sistema de Información Territorial & 30 & 212 \\
\hline 35 & Optativa II & 29 & 365 \\
\hline 36 & Práctica Profesional Supervisada & $24,31,32$ & 365 \\
\hline 37 & Elaboración del Trabajo Final & $24,31,32$ & 365 \\
\hline 38 & Defensa del Trabajo Final & $33,34,35,36,37$ & 45 \\
\hline
\end{tabular}




\section{Resultados}

Los primeros resultados se obtuvieron ya al elaborar el listado de tareas, precedencias y duraciones (Tabla 2). En efecto, se observó que, considerando sólo el régimen de correlatividades y aislando el análisis de otros factores como ser carga horaria total de las asignaturas por cuatrimestre, es posible "adelantar" el cursado y aprobación de algunas asignaturas (esto es, cursarlas y aprobarlas antes de lo que establece el plan de estudios), lo cual estaría hablando de un primer vestigio de flexibilidad del régimen de correlatividades.

Dichas asignaturas son: Introducción a la Ingeniería en Agrimensura (6), Trigonometría (7), Agrimensura Legal I (13) y Geografía Física y Geomorfológica (15).

Al determinar los márgenes totales de las actividades del proyecto, se obtuvieron 6 caminos críticos (los cuales se detallan en la Tabla 3); cada uno de ellos requiere, para ser recorrido, de 1811 días, de donde puede concluirse que la duración mínima de la carrera es cercana a los 5 años calendario (1826 días).

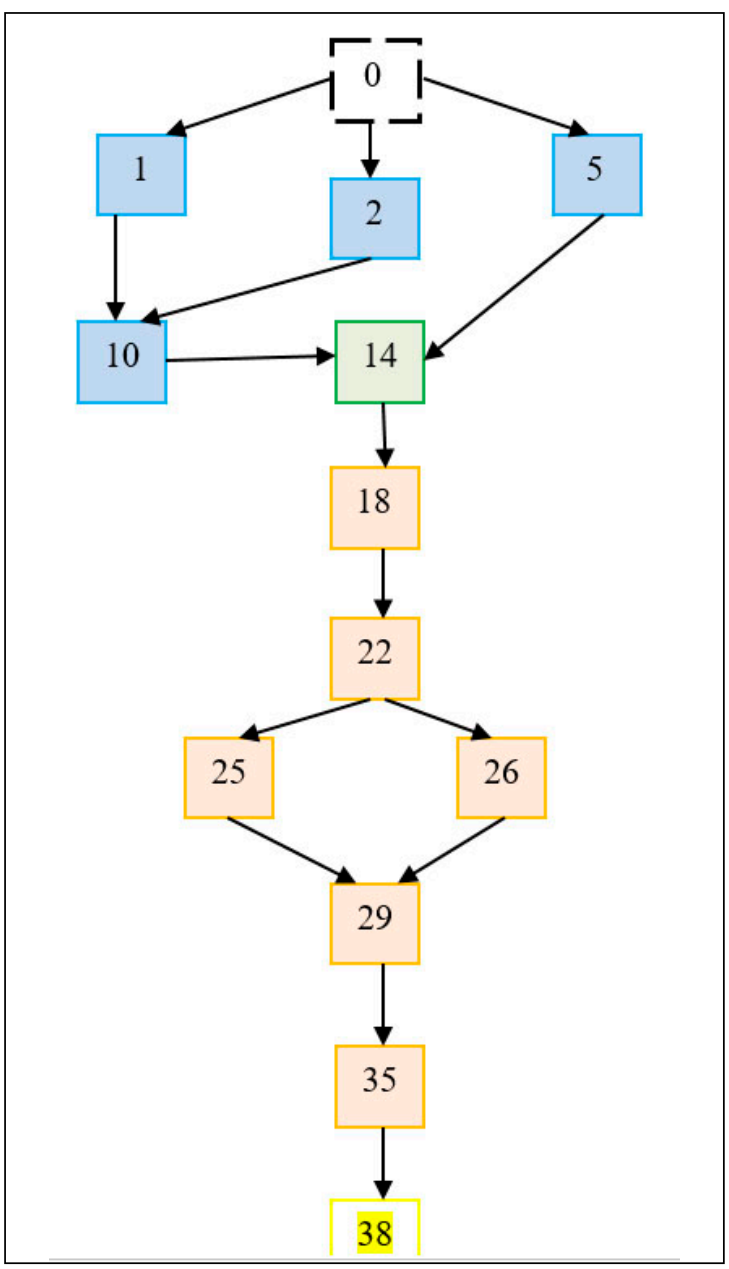

Figura 1: Actividades criticas, segun bloques temáticos a los que pertenecen las asignaturas asociadas

\section{Tabla 3: Tareas y caminos críticos}

\begin{tabular}{|c|c|c|c|c|c|}
\hline \multicolumn{7}{|c|}{ Camino Crítico No } \\
\hline 1 & 2 & 3 & 4 & 5 & 6 \\
\hline Algebra y Geometría Analítica (1) & 1 & CDI1 (2) & 2 & S de R. (5) & 5 \\
\hline Mecánica, Optica y Sonido (10) & 10 & 10 & 10 & 14 & 14 \\
\hline Topografia I (14) & 14 & 14 & 14 & 18 & 18 \\
\hline Topografía II (18) & 18 & 18 & 18 & 22 & 22 \\
\hline Topografia III (22) & 22 & 22 & 22 & 25 & 26 \\
\hline Fotogrametría (25) & GAM (26) & 25 & 26 & 29 & 29 \\
\hline Geografia Física y Geomorfología (29) & 29 & 29 & 29 & 35 & 35 \\
\hline Optativa II (35) & 35 & 35 & 35 & 38 & 38 \\
\hline Defensa del Trabajo Final (38) & 38 & 38 & 38 & & \\
\hline
\end{tabular}


Puede observarse en la Figura 1 que todas las actividades críticas que se relacionan a asignaturas de primer año (1: Álgebra y Geometría Analítica, 2: Cálculo Diferencial e Integral I, 5: Sistemas de Representación y 10: Mecánica, Óptica y Sonido) corresponden al bloque de las Ciencias Básicas (por lo cual se las señalado en azul), mientras que Topografía I (14) — coloreada con verde - es la única asignatura correspondiente al segundo año de estudios y al bloque de Tecnologías Básicas cuya aprobación es crítica en todas las rutas detectadas. Del bloque de Tecnologías Aplicadas (naranja), los alumnos deben priorizar la aprobación de Topografía II y III (ambas del tercer año del plan de estudios), Fotogrametría, Geodesia Astronómica y Matemática y Geodesia Física y Global (de cuarto año) para evitar retrasos en el egreso. Del bloque de Complementarias (rosado), en cambio, resulta crítica la tarea de aprobar Optativa I y, finalmente y por ser la última actividad necesaria para obtener el título de Ingeniero, resulta una tarea crítica la defensa del Trabajo Final (en amarillo por estar vinculada a la formación profesional, y no algún bloque temático de asignaturas).

Dado que en la FACENA las clases de las asignaturas cuatrimestrales se dictan una única vez en cada año lectivo, puede observarse que las asignaturas que pueden recursarse sin retrasar el egreso son aquellas cuya holgura es, al menos, de 1 año. Ellas son: Química General, Informática, Introducción a la Ingeniería en Agrimensura, Agrimensura Legal I, Geografía Física y Geomorfología, Economía y Gestión Empresarial y Optativa I.
Por lo dicho antes, para aquellas que tienen holgura positiva pero menor que un año, puede retrasarse su aprobación, pero no su regularización. En efecto, Ordenamiento Territorial, Mensura y Sistema de Información Territorial tienen margen para retrasar su aprobación hasta 10 meses. En cambio, para egresar en los 5 años propuestos por el plan, la aprobación de Trigonometría, de la Prueba de Traducción Técnica, de Cálculo Diferencial e Integral II, de Electricidad, Magnetismo y Calor, de Estadística y Análisis de Datos, de Teoría de Errores y Compensación, de Fotointerpretación y Teledetección, de Agrimensura Legal II, de Catastro, de Valuaciones Inmobiliarias y de Mediciones Especiales no debe retrasarse más de 5 meses. Finalmente, la aprobación de Dibujo Topográfico y Cartográfico y de Cartografía, así como la realización de la Práctica Profesional Supervisada y la elaboración del Trabajo Final pueden retrasarse, a lo sumo, 1 mes.

\section{Conclusiones}

A partir de los resultados obtenidos, puede concluirse que los trayectos de formación que determinan la duración mínima de la carrera, contienen asignaturas fundamentales para la formación profesional del ingeniero en Agrimensura, pues dichos trayectos están fuertemente vinculados a los bloques de Ciencias Básicas y Tecnologías Aplicadas.

Si bien el plan es bastante flexible en lo que respecta a la aprobación de las asignaturas, no lo es en igual medida en lo que respecta al cursado y regularización de las mismas. 
El hecho de que la regularización y aprobación de Algebra y Geometría Analítica y Cálculo Diferencial e Integral I (ambas asignaturas correspondientes al primer cuatrimestre del primer año de la carrera) resulten actividades críticas, indican que es muy difícil que la duración real de la carrera coincida con la teórica, ya que los menores niveles de rendimiento académico se dan en dicho momento de los estudios, debido a diversos factores entre los que cabe señalar que este período coincide con el proceso de socialización institucional de los alumnos.

\section{REFERENCIAS:}

[1] Caputo, Liliana; Herrmann, Carlos. (2001). Correlatividades "fuertes" y tareas críticas del plan de estudios de la carrera de Bioquímica de la FA.C.E.N.A. (U.N.N.E.). Reunión Anual de Comunicaciones Científicas y Tecnológicas 2001 SECYT (U.N.NE.) Corrientes. Argentina.

[2] Caputo, Liliana. (2005). Profesorado en $\mathrm{Ma}-$ temática de FACENA - UNNE (Plan 2001): Análisis de su plan de estudios por camino crítico. Revista de la Escuela de Perfeccionamiento en Investigación Operativa, No 26. pp 137 - 149. Tandil, Bs. As. ISSN 0329 - 7322.

[3] Galiana, José; Caputo, Liliana. (2006). Plan de estudios del Profesorado en Física de la FACENA - UNNE: Análisis de su régimen de correlatividades por el método de camino crítico. Anales del XIX Encuentro Nac. de Docentes de Investigación Operativa y XVII Escuela de Perfeccionamiento en Investigación Operativa. Rosario (Santa Fe). ISBN 950 -658 -172 - X.

[4] Romero, José; Caputo, Liliana; Porcel, Eduardo; Dapozo, Gladys. (2015). Planes 1999 y 2009 de la Licenciatura en Sistemas de Información de la FACENA - UNNE: Un análisis comparativo mediante CPM. Anales de XXVIII Encuentro Nac. de Docentes en Investigación Operativa, XXVI Escuela de Perfeccionamiento en Investigación Operativa y VIII Red Ibe- roamericana de Evaluación y Decisión Multicriterio. Universidad Nacional del Sur. Bahía Blanca(Buenos Aires).ISBN 978-987-24267-6-7.

[5] Caputo, Liliana; Porcel, Eduardo; Galiana, José; Garau, César. (2008). Descripción del desempeño académico de alumnos y graduados de una carrera universitaria mediante CPM y análisis de conglomerados. Anales del I Encuentro Regional Argentino Brasileño de Investigación Operativa, XXI Encuentro Nacional de Docentes de Investigación Operativa y XIX Escuela de Perfeccionamiento en Investigación Operativa. Posadas (Misiones). ISBN 978 - 987 - 24267 - 0 - 5. [6] Caputo, Liliana; López, Ma. V.; Porcel, Eduardo; Dapozo, Gladys. (2010). Tiempo empleado por los alumnos de la Licenciatura en Sistemas de Información de la FACENA (UNNE), para la obtención del título intermedio de Programador Universitario de Aplicaciones. Anales del II Encuentro Regional Argentino Brasileño de Investigación Operativa, XXIII Encuentro Nacional de Docentes de Investigación Operativa y XXI Escuela de Perfeccionamiento en Investigación Operativa. Tandil (Bs As). ISBN 978 - 2467 - 1 - 2.

[7] Caputo, Liliana; Porcel, Eduardo. (2014). Predicción de la duración de la carrera de Agrimensura de la FACENA-UNNE. Revista de la Escuela de Perfeccionamiento en Investigación Operativa No 36. pp 63 - 80. Tandil (Bs. As.). ISSN 1853 - 9777. 
[8] Caputo, Liliana; Porcel, Eduardo; Avalos, Julián. (2014). Trayectorias de formación de alumnos de las carreras de ingeniería de la FACENA - UNNE. Plan 2001. Actas del XVIII EMCI Nacional y X Internacional. Facultad de Ingeniería. Universidad Nacional de Mar del Plata. ISBN 978- 987- 544 - 564 - 2.

[9] Caputo, Liliana; Porcel, Eduardo; Avalos, Julián. (2014). Ciclo Básico de la Licenciatura en Ciencias Biológicas de FACENA - UNNE: Análisis por CPM del desempeño de sus graduados. Actas de las Cuartas Jornadas de Ingreso y Permanencia en Carreras Científico - Tecnológicas. Facultad de Ciencias Exactas, Ingeniería y Agrimensura. U. Nac. de Rosario. ISBN 978-987-3662 - 01 - 0.

[10] Porcel, Eduardo; Caputo, Liliana; Ramírez Arballo, Ma. (2012). Rendimiento académico en asignaturas de primer año en 2010. Actas de las Terceras Jornadas de Ingreso y Permanencia en Carreras Científico - Tecnológicas. Facultad de Ingeniería. Universidad Nacional de San Juan. ISBN 978 - 950 - 605- 719 - 0.

[11] Caputo, Liliana; Porcel, Eduardo; Lucero, Irma. (2016). Desempeño de estudiantes universitarios de carreras de Ingeniería. Su análisis mediante la técnica de datos composicionales. Anales del XXIX Encuentro Nacional de Docentes de Investigación Operativa y XXVII Escuela de Perfeccionamiento en Investigación Operativa. Facultades de Ciencias Económicas y de Ciencias Físicomatemáticas e Ingeniería de la Universidad Católica Argentina.CABA.ISBN 978-987-24267-7-4.

[12] Romero, José; Caputo, Liliana; Porcel, Eduardo; Ferrari, Elvira. (2016). Análisis de datos composicionales para el monitoreo del desempeño de estudiantes de una carrera universitaria de grado. Revista Extensionismo, Innovación y Transferencia Tecnológica. Claves para el de- sarrollo. Volumen 3. pp 104 - 112. Facultad de Ciencias Exactas y Naturales y Agrimensura - UNNE. Corrientes. ISSN 2422 - 6424. [13] Porcel, Eduardo; López, Ma.V.; Dapozo, Gladys; Caputo, Liliana. (2010). Probabilidad de aprobar las asignaturas de las carreras de Ingeniería Eléctrica e Ingeniería Electrónica de la FACENA (UNNE). Revista de la Escuela de Perfeccionamiento en Investigación Operativa No 31. pp 101 - 118. Tandil (Bs. As.). ISSN 0329 - 7322.

[14] Avalos Julián; Caputo, Liliana; Porcel, Eduardo. (2012). Tiempos de aprobación de asignaturas del Ciclo Básico de Ingeniería en Electrónica de FACENA. Anales del XXV Encuentro Nacional de Docentes de Investigación Operativa y XXIII Escuela de Perfeccionamiento en Investigación Operativa. Facultad de Ingeniería. UBA. CABA. ISBN 978 - 24267 - 3 - 6 .

[15] Pérez Mackeprang, Carlos; Alberto, Catalina; Carignagno, Claudia.; Castro, Sergio. (1998). Programación por camino crítico, introducción al método: actividades en los vértices. Revista de la Escuela de Perfeccionamiento en Investigación Operativa No 14. pp. 15 - 28. Tandil (Bs. As.). ISSN 0329 - 7322. [16] Caputo, Liliana. (2007). C.P.M.: Su aplicación para el análisis de planes de estudios de carreras del Nivel Superior. Primera Parte: Uso de Redes de Actividades Ciertas. Revista de la Escuela de Perfeccionamiento en Investigación Operativa No 28 -pp.170-188.Tandil(Bs.As.).ISSN 0329-7322.

[17] Caputo, Liliana; Galiana, José. (2008). C.P.M.: Su aplicación para el análisis de planes de estudios de carreras del Nivel Superior. Segunda Parte: Uso de Redes de Actividades Estocásticas. Revista de la Escuela de Perfeccionamiento en Investigación Operativa No 29. pp. 101 - 118. Tandil (Bs. As.). ISSN 0329 - 7322. 